OPEN ACCESS

Edited by:

Kayo Masuko,

Sanno Medical Center, Japan

Reviewed by:

Takahisa Gono

Nippon Medical School, Japan

Tue Wenzel Kragstrup,

Aarhus University, Denmark

Marika Tardella,

Università Politecnica delle

Marche, Italy

*Correspondence:

Yudong Liu

yudongliu1983@126.com

tThese authors have contributed equally to this work

Specialty section

This article was submitted to

Rheumatology,

a section of the journal

Frontiers in Medicine

Received: 08 August 2021

Accepted: 30 September 2021

Published: 26 October 2021

Citation:

Liang L, Chen J, Di C, Zhan M, Bao H,

Xia C, Fan C and Liu Y (2021) Serum

Human Epididymis Protein 4 as a

Novel Biomarker in Identifying Patients

With Interstitial Lung Disease in

Rheumatoid Arthritis.

Front. Med. 8:755268.

doi: 10.3389/fmed.2021.755268

\section{Serum Human Epididymis Protein 4 as a Novel Biomarker in Identifying Patients With Interstitial Lung Disease in Rheumatoid Arthritis}

\author{
Liu Liang ${ }^{1,2 t}$, Jiali Chen ${ }^{3 t}$, Chun Di $i^{1+}$, Minghua Zhan ${ }^{1}$, Huizhang Bao ${ }^{1}$, Changsheng Xia ${ }^{1}$, \\ Chunhong Fan ${ }^{1}$ and Yudong Liu ${ }^{1 *}$
}

\footnotetext{
${ }^{1}$ Department of Clinical Laboratory, Peking University People's Hospital, Beijing, China, ${ }^{2}$ Department of Clinical Laboratory, The Second People's Hospital of Qinzhou, Qinzhou, China, ${ }^{3}$ Department of Rheumatology and Immunology, The Second Xiangya Hospital, Central South University, Changsha, China
}

Objective: Human epididymis protein 4 (HE4) have been implicated in the pulmonary involvements. We aimed to investigate the clinical utility of HE4 in clinical stratification in patients with rheumatoid arthritis (RA).

Methods: This study included a discovery cohort comprising 70 RA patients and 64 healthy controls $(\mathrm{HCs})$, and a validation cohort comprising $98 \mathrm{RA}$ patients and $75 \mathrm{HCs}$. Human epididymis protein 4 were determined by electrochemical luminescence analyzer.

Results: The levels of HE4 were significantly elevated in patients with RA compared to HCs. The positive rates of HE4 in patients with RA and HCs were $50.0 \%$ and 0 , respectively, in the discovery cohort and 53.1 and $1.3 \%$, respectively, in the validation cohort. When RA patients were subgrouped according to HE4 status, HE4positive group displayed higher prevalence of interstitial lung disease (ILD) compared to HE4-negative group (28.6 vs. $11.4 \%$ in discovery cohort and 57.7 vs. $8.7 \%$ in the validation cohort). A positive correlation between the levels of HE4 with the degree of lung impairment was identified. Receiver operating curve $(\mathrm{ROC})$ analysis revealed an optimal cut-off value of 104.3 pmol/L in HE4 for distinguishing RA-ILD from RA-non ILD with the areas under the curve $(A \cup C)$ of 0.790 . Multivariate logistic regression analysis illustrated that high levels of HE4 independently identified patients with RA-ILD (OR, 9.080, $p<0.001)$.

Conclusion: Our findings showed a novel role of HE4 in RA risk stratification, suggest that introducing HE4 to the current RA test panel may serve as an indicator in identifying RA patients for further RA-ILD workups, such as high-resolution computed tomography (HRCT).

Keywords: rheumatoid arthritis, human epididymis protein 4, interstitial lung disease, risk stratification, biomarker 


\section{INTRODUCTION}

Rheumatoid arthritis (RA) is a chronic and progressive autoimmune joint disorder characterized by synovial inflammation, joint destruction, and various extra-articular manifestations (EAMs) (1-3). Pulmonary complications, particularly interstitial lung disease (ILD), represent an important extra-articular feature of various EAMs and a major cause of mortality in patients with RA (2-5). A recent study showed that $72 \%$ patients with RA-ILD had an inpatient admission and $76 \%$ had an emergency room visit (6). Further, clinically significant RA-ILD accounts for $10 \%$ of the RA population, and is associated with shortened survival and more severe underlying disease (7). To date, there are few treatments that are proven to be effective in the treatment of RA-ILD, and biomarkers that can predict RA patients at risk for ILD are in great need.

Human epididymis protein 4 (HE4), a human epididymisspecific protein, has been widely utilized in clinical practice as a tumor marker in identifying patients at risk of ovarian cancer (8). Human epididymis protein 4 is expressed in multiple tissues in the oral cavity, the respiratory tracts as well as in renal tubular epithelial cells (9). Previous studies have shown that serum HE4 levels were significantly elevated in patients with systemic sclerosis (SSc)-ILD (10). Further, high levels of HE4 can stratify patients into SSc-ILD subsets (10). We also showed that HE4 can identify primary Sjögren's syndrome (pSS) patients at risk of pulmonary/renal involvements (11). Taken together, these findings suggest that HE4 may have a diagnostic potential in disease stratification in patients with autoimmune diseases. In this study, we aimed to investigate the clinical utility of HE4 in the diagnosis and disease stratification in RA.

\section{MATERIALS AND METHODS}

\section{Subjects}

To evaluate the clinical utility of HE4 in RA patients, we recruited two cohorts of RA patients, including the discovery cohort and the validation cohort (Table 1). The discovery cohort retrospectively collected the clinical data of 70 consecutive RA inpatients who were tested for the female-tumor biomarker screening panel from Jan. 2020 to Sep. 2020 and 64 consecutive healthy controls (HCs) who went to Peking University People's Hospital (PKUPH) for annual physical examination and were tested for the female-tumor biomarker screening panel between Jan. 2020 and Oct. 2020. To verify the clinical relevance of HE4 for RA patients, we prospectively recruited 98 RA inpatients and $75 \mathrm{HCs}$ and regarded those patients as the validation cohort. All RA patients were diagnosed according to 2010 American College of Rheumatology (ACR)/European League Against Rheumatism classification criteria (12). Rheumatoid Arthritis patients with cancer or history of malignant neoplasm were excluded. Healthy controls who displayed abnormality in blood differential tests, biochemistry profile tests, autoantibody profile tests or had history of systemic

TABLE 1 | Demographic and clinical characteristics between patients with rheumatoid arthritis (RA) and healthy controls (HCs).

\begin{tabular}{|c|c|c|c|c|c|c|}
\hline & \multicolumn{3}{|c|}{ Discovery cohort } & \multicolumn{3}{|c|}{ Validation cohort } \\
\hline Age, years & $62(53,70)$ & $40(31,49)$ & $<0.001$ & $64(55,71)$ & $60(52,69)$ & 0.154 \\
\hline Gender, female & 70 (100.0) & $64(100.0)$ & - & 77 (78.6) & 55 (73.3) & 0.259 \\
\hline Smoking status & $2(2.9)$ & - & - & $5(5.1)$ & - & - \\
\hline TJC28 & $4(1,14)$ & & & $8(1,16)$ & & \\
\hline DAS28-ESR & $5.4(3.9,7.2)$ & & & $5.2(3.7,6.8)$ & & \\
\hline Anti-CCP, positive & $60(85.7)$ & & & $87(88.8)$ & & \\
\hline RF, positive & $52(74.3)$ & & & 79 (80.6) & & \\
\hline Interstitial lung disease & $14(20.0)$ & & & $34(34.7)$ & & \\
\hline NSAIDs & $20(28.6)$ & & & 16 (16.3) & & \\
\hline Steroids & $18(25.7)$ & & & $32(32.7)$ & & \\
\hline cDMARDs & $52(74.3)$ & & & $60(61.2)$ & & \\
\hline bDMARDs & $10(14.3)$ & & & $11(11.2)$ & & \\
\hline
\end{tabular}

Data are presented as median (interquartile range) and n (\%). Anti-CCP, anti-cyclic citrullinated peptide antibody; bDMARDs, biological disease modifying anti-rheumatic drugs; cDMARDs,

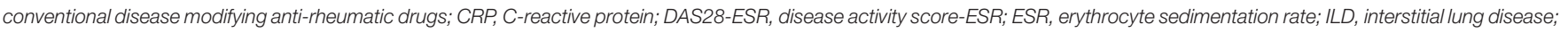
NSAIDs, non-steroid anti-inflammatory drugs; RF, rheumatoid factor; SJC28, swollen joint counts in 28 joints; TJC28, tender joint counts in 28 joints. 
TABLE 2 | Levels of positive tumor markers between patients with rheumatoid arthritis (RA) and healthy controls (HCs).

\begin{tabular}{|c|c|c|c|c|c|c|c|}
\hline Positive biomarkers & Cut-off value & \multicolumn{3}{|c|}{ Discovery cohort } & \multicolumn{3}{|c|}{ Validation cohort } \\
\hline CEA, $n(\%)$ & $4.7 \mathrm{ng} / \mathrm{ml}$ & $9(12.9)$ & $0(0.0)$ & 0.003 & & & \\
\hline AFP, $n(\%)$ & $26.4 \mathrm{ng} / \mathrm{ml}$ & $1(1.4)$ & $3(4.7)$ & 0.348 & & & \\
\hline CA19-9, n (\%) & $39 \mathrm{U} / \mathrm{ml}$ & $8(11.4)$ & $1(1.6)$ & 0.035 & & & \\
\hline CA15-3, n (\%) & $26.4 \mathrm{U} / \mathrm{ml}$ & $4(5.7)$ & $0(0.0)$ & 0.121 & & & \\
\hline CYFRA21-1, $n$ (\%) & $3.3 \mathrm{ng} / \mathrm{ml}$ & $15(21.4)$ & $8(12.5)$ & 0.251 & & & \\
\hline NSE, $n(\%)$ & $16.3 \mathrm{ng} / \mathrm{ml}$ & $2(2.9)$ & $7(10.9)$ & 0.086 & & & \\
\hline ProGRP, $n(\%)$ & $68.3 \mathrm{pg} / \mathrm{ml}$ & $10(14.3)$ & $0(0.0)$ & 0.002 & & & \\
\hline SCC, $n(\%)$ & $2.7 \mathrm{ng} / \mathrm{ml}$ & $4(5.7)$ & $2(3.1)$ & 0.682 & & & \\
\hline $\mathrm{HE} 4, n(\%)$ & $76.2 \mathrm{pmol} / \mathrm{L}$ (<60 years) & 7/31 (22.6) & 0/61 (0.0) & $<0.001$ & 13/34 (38.2) & $0 / 37(0.0)$ & $<0.001$ \\
\hline
\end{tabular}

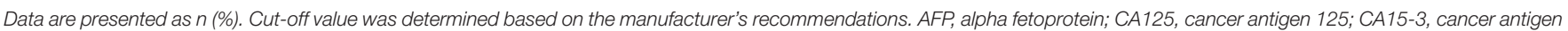

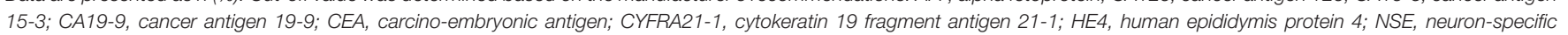
enolase; ProGRP, progastrin-releasing peptide; SCC, squamous cell carcinoma antigen.

A

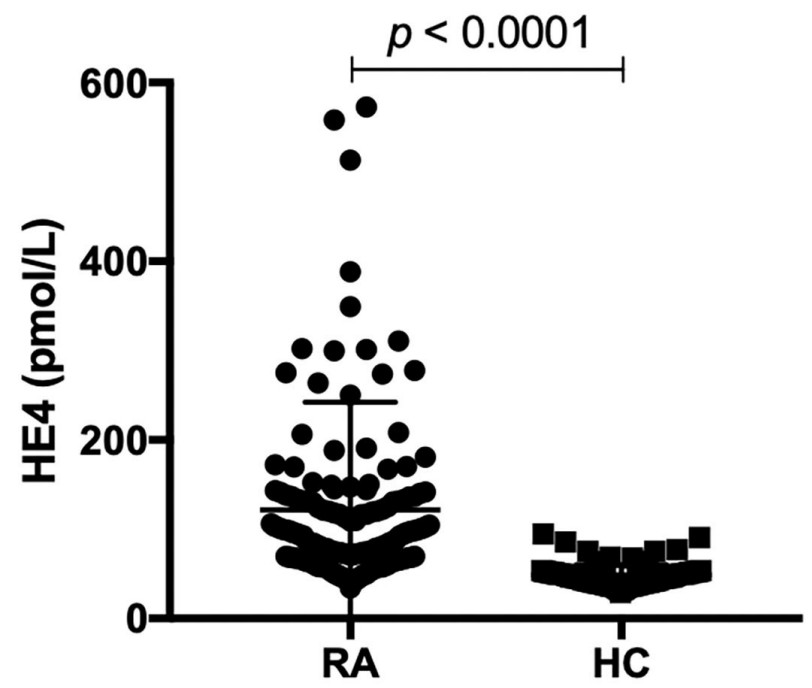

B

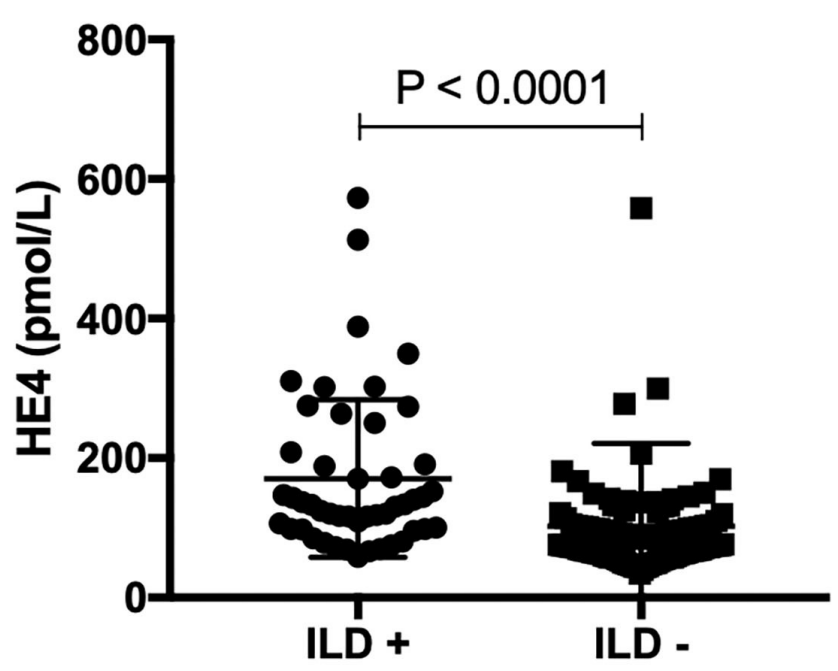

FIGURE 1 | (A) Levels of human epididymis protein 4 (HE4) were elevated in patients with rheumatoid arthritis (RA) $(n=168)$ compared to healthy controls (HCs) $(n=$ 139). Associations between levels of HE4 with (B) Levels of human epididymis protein 4 (HE4) were elevated in rheumatoid arthritis (RA) patients with interstitial lung disease (ILD) $(n=48)$ compared to RA patients without ILD $(n=120)$.

diseases, neoplastic, and autoimmune/autoinflammatory diseases were excluded. The study protocol was reviewed and approved by the Ethical Committee of PKUPH (Protocol number: 2019PHB244). Informed consents of discovery cohort were waived for those patients in the discovery cohort. However, all participants in the validation cohort gave written informed consent.

\section{Data Collection}

Demographic features and clinical and laboratory findings, including age, disease duration, swollen joint count in 28 joints (SJC28), tender joint count in 28 joints (TJC28), erythrocyte sedimentation rate (ESR), C-reactive protein (CRP), rheumatoid factor (RF), anti-CCP antibodies, EAMs, treatments and medical history, were collected from the medical database of PKUPH. 
A

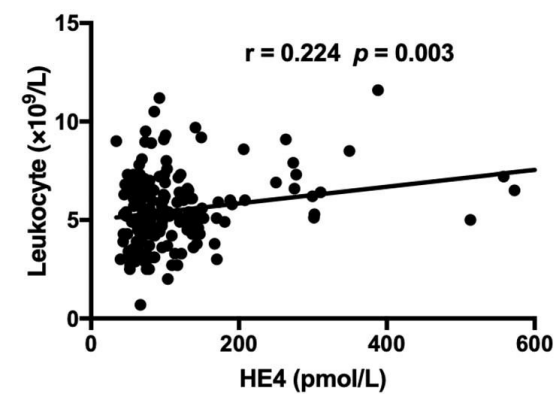

D

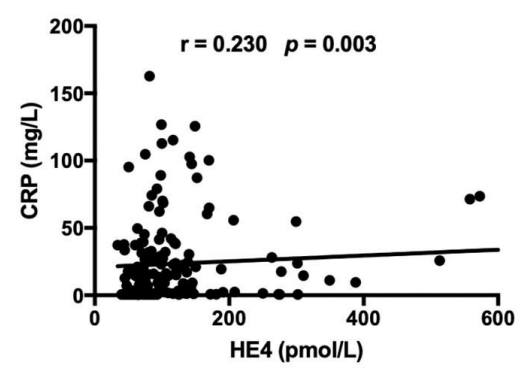

B

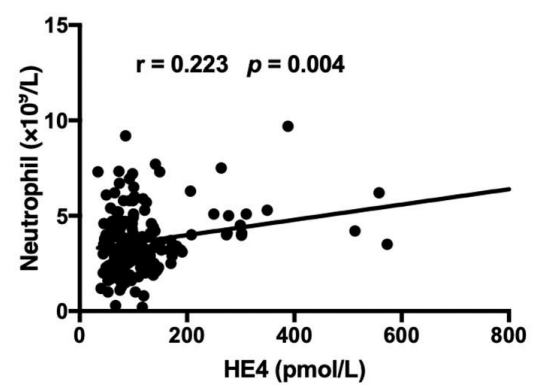

E

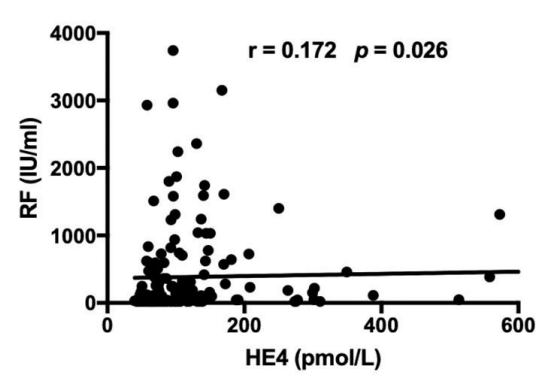

C

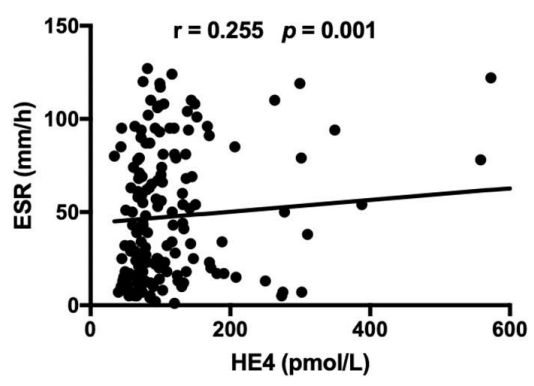

$\mathbf{F}$

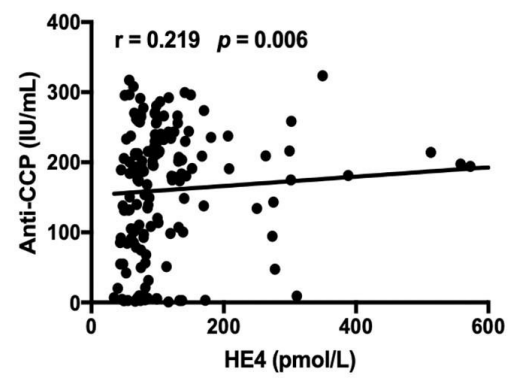

FIGURE 2 | Associations between levels of HE4 with (A) total leukocyte count, (B) total neutrophil count, (C) erythrocyte sedimentation rate (ESR), (D) C-reactive Protein (CRP), (E) Rheumatoid factor, and (F) anti-CCP antibodies ( $n=168)$.

Disease Activity Score (DAS28) was assessed as previous described $(13,14)$.

\section{Serum Tumor Biomarkers Determination}

All the tumor biomarkers in the female-tumor biomarker screening panel, including alpha fetoprotein (AFP), carcinoembryonic antigen (CEA), neuron-specific enolase (NSE), cytokeratin 19 fragment antigen 21-1 (CYFRA21-1), cancer antigen 125 (CA125), CA15-3, CA19-9, progastrinreleasing peptide (ProGRP), squamous cell carcinoma (SCC), and HE4 were determined by Roche Cobas electrochemical luminescence analyzer (Hoffmann-La Roche AG., Basel, Switzerland), according to the manufacturer's instructions. The cut-off values for positivity of these tumor markers were determined based on the manufacturer's recommendations.

\section{Assessment of Interstitial Lung Disease}

The assessment of ILD was performed by two pulmonologists and two radiologists with more than 10 years of thoracic imaging experience, mainly based on the symptoms and respective abnormalities suggestive of ILD in high-resolution computed tomography (HRCT) and pulmonary function tests (PFTs). The RA-ILD was assessed semi-quantitatively based on the HRCT scans, and any indeterminate ILD were excluded from the analyses. Pulmonary function tests were determined as forced vital capacity (FVC), the median forced expiratory volume in 1 second (FEV1), and diffusing capacity of the lung for carbon monoxide (DLco). Forced vital capacity, FEV1, and DLco were presented as a percentage of the predicted values for the patient's age, sex, and height, as previously described (15). Abnormalities of PFTs were defined as predicted values of FVC $<80 \%$ and DLco $<$ $70 \%(16)$.

\section{Statistical Analysis}

Continuous variables were presented as mean \pm standard deviation (SD) for normal distribution or median (interquartile range, IQR) for abnormal distribution. Categorical variables were shown as numbers (percentages) of the total samples. The statistical significance between groups was assessed using the Mann-Whitney U-test, Student $t$-test, Chi-square $\left(\chi^{2}\right)$ test, where it was applicable. Spearman's correlation test was used to determine the relationships between HE4 and clinical parameters. The receiver operating characteristic (ROC) curve was generated to evaluate the sensitivity, specificity and areas under the ROC curve (AUC) with the 95\% confidence interval ( $95 \%$ CI). The optimal cutoff value for predicting the incidence of ILD was identified by calculating the Youden index. Multivariate Logistic regression analyses were used to determine the risk factors for ILD. Once a univariate statistic was generated, the multivariate model was then built using a forward selection procedure. Variables with a $p$-value of $<0.1$ in the univariate analysis were first considered as candidates for the multivariate model, then variables with a $p$-value of $<0.05$ were used in the final model, and odds ratios (ORs) were calculated with 95\% CI. Data analyses were calculated using SPSS 20.0 statistical software 
TABLE 3 | Clinical characteristics of patients with rheumatoid arthritis (RA) according to HE4 status*.

\begin{tabular}{|c|c|c|c|c|c|c|}
\hline & \multicolumn{3}{|c|}{ Discovery cohort } & \multicolumn{3}{|c|}{ Validation cohort } \\
\hline & $\begin{array}{c}\text { HE4+ } \\
(n=35)\end{array}$ & $\begin{array}{c}\text { HE4- } \\
(n=35)\end{array}$ & $p$-value & $\begin{array}{c}\text { HE4+ } \\
(n=52)\end{array}$ & $\begin{array}{c}\text { HE4- } \\
(n=46)\end{array}$ & $p$-value \\
\hline Age, years, median (IQR) & $69(62,77)$ & $56(50,62)$ & $<0.001$ & $67(56,74)$ & $61(54,67)$ & 0.427 \\
\hline Gender, female, $n(\%)$ & $35(100.0)$ & 35 (100.0) & - & $38(73.1)$ & $39(84.8)$ & 0.159 \\
\hline Smoking status & $0(0.0)$ & $2(5.7)$ & 0.493 & $5(9.6)$ & $0(0.0)$ & 0.058 \\
\hline Duration, years, median (IQR) & $9(3,18)$ & $8(3,16)$ & 0.306 & $12(9,18)$ & $8(3,21)$ & 0.210 \\
\hline SJC28, median (IQR) & $2(1,10)$ & $2(0,6)$ & 0.245 & $2(0,5)$ & $7(2,11)$ & 0.008 \\
\hline TJC28, median (IQR) & $4(1,4)$ & $4(2,12)$ & 0.831 & $6(1,17)$ & $8(3,16)$ & 0.313 \\
\hline ESR, mm/h, median (IQR) & $66(25,93)$ & $39(20,62)$ & 0.038 & $53(24,80)$ & $43(16,76)$ & 0.230 \\
\hline CRP, mg/L, median (IQR) & $14.6(4.3,42.0)$ & $10.7(2.1,29.1)$ & 0.180 & $16.2(5.5,28.6)$ & $5.6(0.6,37.5)$ & 0.021 \\
\hline DAS28-ESR, median (IQR) & $5.77(4.61,8.04)$ & $5.54(4.11,6.85)$ & 0.226 & $5.84(3.81,6.85)$ & $6.58(5.24,8.24)$ & 0.196 \\
\hline RF, positive, $n(\%)$ & $27(77.1)$ & $25(71.4)$ & 0.584 & $46(88.5)$ & $33(71.7)$ & 0.037 \\
\hline RF, positive, IU/ml & $254.0(79.7,729.5)$ & $29.9(22.8,138.4)$ & 0.004 & $201.0(44.1,960.3)$ & $63.8(20.0,298.0)$ & 0.008 \\
\hline Anti-CCP, positive, $n(\%)$ & $31(88.6)$ & $29(82.9)$ & 0.495 & $46(88.5)$ & $41(89.1)$ & 0.917 \\
\hline Anti-CCP, positive, U/ml & $225.6(173.4,257.4)$ & $167.3(64.2,229.5)$ & 0.734 & $199.4(147.3,234.6)$ & $136.4(83.0,196.5)$ & 0.004 \\
\hline NLR, median (IQR) & $3.0(2.04,4.46)$ & $2.71(1.79,3.97)$ & 0.262 & $2.54(1.87,4.56)$ & $2.56(1.86,3.65)$ & 0.184 \\
\hline Platelets, $10^{9} / \mathrm{L}$, median (IQR) & $281(213,339)$ & $280(220,320)$ & 0.944 & $225(178,296)$ & $242(198,310)$ & 0.438 \\
\hline Hemoglobin, g/L, median (IQR) & $103(95,116)$ & $114(101,121)$ & 0.165 & $105(94,121)$ & $115(97,132)$ & 0.300 \\
\hline C3, g/L, median (IQR) & $0.99(0.86,1.18)$ & $0.99(0.89,1.19)$ & 0.874 & $0.92(0.80,1.03)$ & $1.09(0.88,1.21)$ & 0.017 \\
\hline C4, g/L, median (IQR) & $0.19(0.14,0.25)$ & $0.21(0.17,0.26)$ & 0.585 & $0.22(0.17,0.26)$ & $0.21(0.18,0.25)$ & 0.078 \\
\hline IgG, g/L, median (IQR) & $13.3(9.7,15.9)$ & $13.9(11.4,19.0)$ & 0.224 & $12.9(8.9,16.9)$ & $15.1(11.1,17.9)$ & 0.298 \\
\hline Interstitial lung disease, $n$ (\%) & $10(28.6)$ & $4(11.4)$ & 0.073 & $30(57.7)$ & $4(8.7)$ & $<0.001$ \\
\hline Leukocytopenia, $n$ (\%) & $4(11.4)$ & $6(17.1)$ & 0.495 & $3(5.8)$ & $10(21.7)$ & 0.020 \\
\hline Anemia, $n(\%)$ & $22(62.9)$ & $20(57.1)$ & 0.626 & $32(61.5)$ & $20(43.5)$ & 0.115 \\
\hline Sjögren's syndrome, $n$ (\%) & $1(2.9)$ & $0(0.0)$ & 1.000 & $21(40.4)$ & $17(37.0)$ & 0.728 \\
\hline \multicolumn{7}{|l|}{ Medications } \\
\hline NSAIDs, $n(\%)$ & $13(37.1)$ & $7(20.0)$ & 0.112 & $8(15.4)$ & $8(17.4)$ & 0.530 \\
\hline Steroids, $n$ (\%) & $11(31.4)$ & $7(20.0)$ & 0.274 & 18 (34.6) & $14(30.4)$ & 0.802 \\
\hline cDMARDs, $n(\%)$ & $27(77.1)$ & 25 (71.4) & 0.584 & 33 (63.5) & 27 (58.7) & 0.709 \\
\hline bDMARDs, $n(\%)$ & $3(8.6)$ & $7(20.0)$ & 0.172 & $5(9.6)$ & $6(13.0)$ & 0.517 \\
\hline
\end{tabular}

*The reference range of human epididymis protein 4 (HE4) was determined based on the manufacturer's recommendations. Data are presented as median (Interquartile Range) and $n$ (\%). Statistical significance was determined by Mann-Whitney U test and Chi-square ( $\chi 2$ ) test. IQR, interquartile range; Anti-CCP, anti-cyclic citrullinated peptide antibody; bDMARDs, biological disease modifying anti-rheumatic drugs; CDMARDs, conventional disease modifying anti-rheumatic drugs; CRP, C-reactive protein; DAS28-ESR, disease activity score-ESR; ESR, erythrocyte sedimentation rate; ILD, interstitial lung disease; NLR, neutrophil/lymphocyte ratio; NSAIDs, non-steroid anti-inflammatory drugs; RF, rheumatoid factor; SJC28, swollen joint counts in 28 joints; TJC28, tender joint counts in 28 joints.

package (SPSS Inc., Chicago, Illinois, USA) or GraphPad Prism 8 (GraphPad Software Inc.). A significant difference was defined as $p<0.05$.

\section{RESULTS}

\section{Characteristics of Patients With RA in the Two Cohort}

Detailed clinical and laboratory characteristics of the two cohort are presented in Table 1. The median duration of RA patients was 8.5 years for the discovery cohort and 9.5 years for the validation cohort. The median DAS28 was 5.4 (IQR 3.9, 7.2) for RA patients in the discovery cohort and 5.2 (IQR 3.7, 6.8) for RA patients in the validation cohort. Anti-CCP antibodies were positive in $85.7 \%$ of RA patients in the discovery cohort and $88.8 \%$ of RA patients in the validation cohort. Rheumatoid factor was positive in $74.3 \%$ of RA patients in the discovery cohort and $80.6 \%$ of RA patients in the validation cohort. The prevalence of RA-ILD was $20.0 \%$ in the discovery cohort and $34.7 \%$ in the validation cohort, respectively.

\section{The Levels of HE4 Were Significantly Elevated in Patients With RA}

The cut-off values for positivity of all tumor markers in the female-tumor biomarker screening panel are listed in Table 2. Compared to other tumor markers, HE4 displayed the highest positive rates in RA patients (50.0\%), followed by CYFRA21-1 (21.4\%) and CA125 (20.0\%) (Table 2). No HCs were detected positive for HE4. Of interest, the prevalence of HE4 in RA patients $<60$ years old, RA patients aged between 60 and 70 years and RA patients over 70 years old were 22.6, 66.7, and $77.8 \%$, respectively. 
$\mathbf{A}$

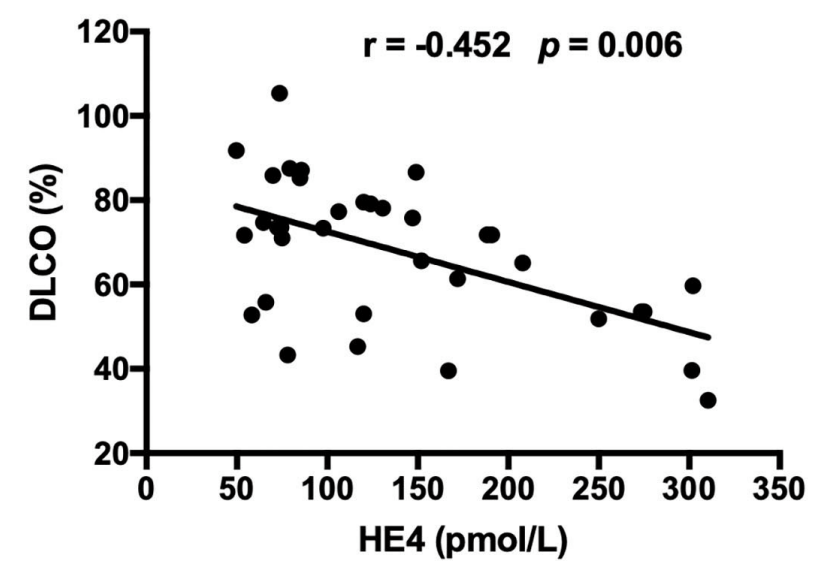

B

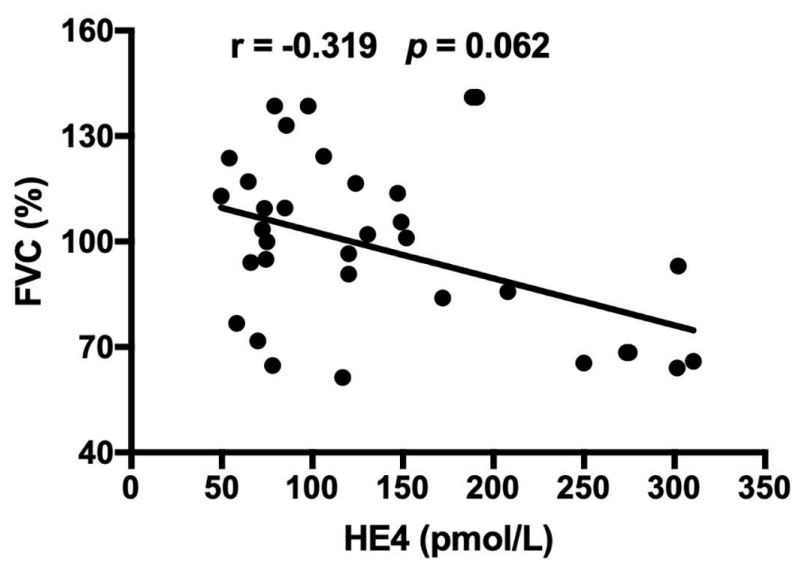

FIGURE 3 | (A) Associations between levels of HE4 and diffusing capacity of the lung for carbon monoxide (DLco) which was presented as a percentage of the predicted values for the patient's age, sex, and height $(n=35)$. (B) Associations between levels of HE4 and forced vital capacity (FVC), which was presented as a percentage of the predicted values for the patient's age, sex, and height $(n=35)$.

Since HE4 was the most significant marker among all the tumor markers in female-tumor biomarker screening panel, we next focus the clinical performance of HE4 in RA. We first verified these results in the validation cohort. The positive rate of HE4 was $53.1 \%$ in this cohort, which was similar to the discovery cohort (Table 2). A similar trend was also observed in RA patients in the validation cohort.

The levels of HE4 were significantly elevated in patients with RA compared to those in HCs (Figure 1A). Further, patients with RA-ILD exhibited significantly higher levels of HE4 compared to patients without RA-ILD ( $p<0.0001$ ) (Figure 1B). The levels of HE4 were significantly associated with total leukocytes number $(p$ $=0.003)$, total neutrophil number $(p=0.003), \operatorname{ESR}(p=0.001)$, CRP $(p=0.003)$, RF $(p=0.026)$, and anti-CCP $(p=0.006)$ (Figures 2A-F).

\section{Characteristics of Patients With RA According to HE4 Levels}

Clinical and laboratory characteristics were compared between HE4-positive RA patients and HE4-negative RA patients. Patients in the HE4-positive group displayed similar disease duration and DAS28-ESR (Table 3). Further, HE4-positive patients showed similar laboratory characteristics, including RF positivity, anti-CCP positivity, levels of C3, levels of $\mathrm{C} 4$, and levels of IgG (Table 3). Of interest, the prevalence of RA-ILD in patients positive for $\mathrm{HE} 4$ was twice as much as that in patients negative for HE4 (28.6 vs. 11.4\%, $p=0.073$ ) (Table 3). The higher incidence of RA-ILD in HE4-positive RA was also confirmed in the validation cohort ( 57.7 vs. $8.7 \%, p<0.001$ ). Consistent with higher levels of HE4 in patients with RA-ILD, the levels of HE4 displayed a significantly negative correlation with DLco\% $(r=-0.452, p=0.006)$ (Figure 3A). In addition, a negative correlation between the levels of HE4 and FVC\% $(r=-0.319$, $p=0.062$ ) was noticed (Figure 3B). In contrast, no significant
TABLE 4 | Associations between levels of rheumatoid factor (RF) or anti-cyclic citrullinated peptide antibody (Anti-CCP) and diffusing capacity of the lung for carbon monoxide (DLCo) or forced vital capacity (FVC) in rheumatoid arthritis (RA) patients with interstitial lung disease (ILD)*.

\begin{tabular}{lccccc}
\hline & \multicolumn{2}{c}{ Anti-CCP } & & \multicolumn{2}{c}{ RF } \\
\cline { 2 - 3 } \cline { 5 - 6 } & $\mathbf{r}$ & $\boldsymbol{p}$-value & & $\mathbf{r}$ & $\boldsymbol{p}$-value \\
\hline FVC\% & -0.242 & 0.198 & & 0.059 & 0.756 \\
DLCO\% & -0.059 & 0.756 & & 0.094 & 0.621 \\
\hline
\end{tabular}

${ }^{*} D L C O$ or FVC was presented as a percentage of the predicted values for the patient's age, sex, and height. $r$ was determined by Spearman's correlation test.

correlations between RF or anti-CCP and DLco\% or FVC\% were identified (Table 4).

\section{Diagnostic Potential of HE4 in RA-ILD}

Receiver operating curve (ROC) analysis was utilized to characterize the clinical performance of HE4 in identifying patients with RA-ILD (Figure 4). Based on Youden index, the optimal cut-off value of HE4 for distinguishing RA-ILD from RAnon ILD was $104.3 \mathrm{pmol} / \mathrm{L}$ with an area under the curve (AUC) of 0.790 and a sensitivity and a specificity of 70.8 and $77.5 \%$, respectively. In addition, ROC analysis was also performed to calculate the cut-off values of high-CCP and high-RF, and the optimal levels were $133.14 \mathrm{U} / \mathrm{ml}$ and $170.5 \mathrm{IU} / \mathrm{ml}$, respectively. These cut-off values were further used to identify which RA patients are at risk of having ILD (Table 5). Univariable analysis showed that male gender $(p=0.002)$, old age $(p=0.012)$, and patients positive for HE4 $(p<0.001)$ were risk factor in predicting RA-ILD. In contrast, RA-non ILD patients tended to show higher SJC28 $(p=0.024)$ and TJC28 $(p=0.002)$ compare to patients with RA-ILD. Besides, patients with ILD had higher percentages of high-RF and high-anti-CCP than those without, 


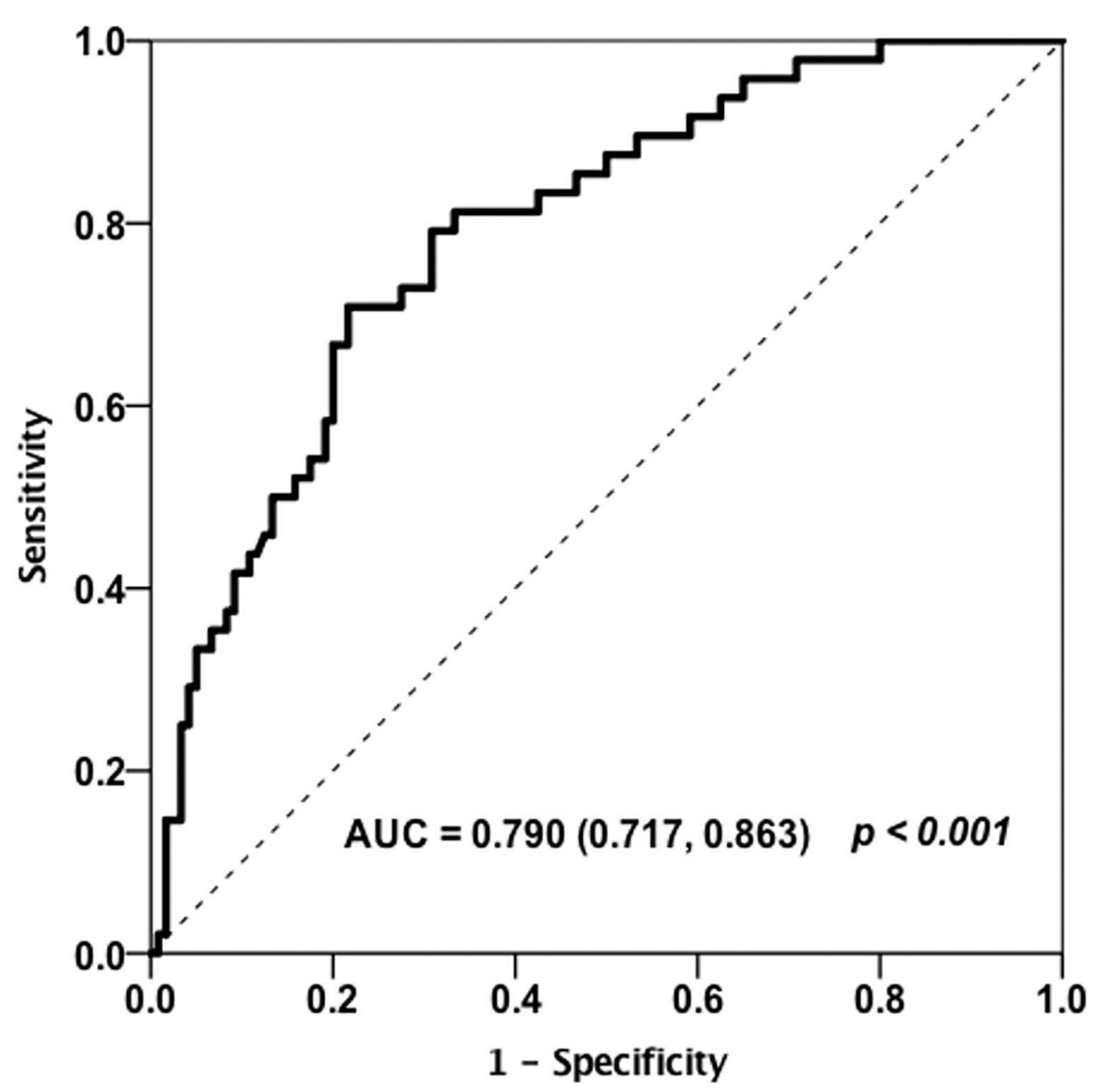

FIGURE 4 | Receiver operating curve (ROC) analysis on the clinical performance of HE4 in identifying patients with RA-ILD ( $n=168)$.

but the differences were insignificant. To further assess the independent predictors for developing RA-ILD, a multivariate logistic regression analysis was performed. Of note, female gender (OR, 0.275; 95\%CI, 0.085-0.893; $p=0.032$ ), DAS28-ESR (OR, 0.821; 95\%CI, 0.690-0.976; $p=0.026$ ), and high levels of HE4 (OR, 9.080; 95\%CI, 3.481-23.682; $p<0.001$ ) persisted as independent risk factors for predicting RA-ILD (Table 5).

\section{DISCUSSION}

Human epididymis protein 4 has been widely utilized as an effective biomarker in the diagnosis and follow-up of patients with ovarian cancer. Of interest, accumulating evidence suggest that HE4 may also has diagnostic potential in other clinical settings, including lung adenocarcinomas (17), renal fibrosis (18), cystic fibrosis (19), as well as autoimmunerelated organ involvements, such as SSc-ILD (10) and pSSrelated pulmonary/renal involvements (11). In this study, we extended the clinical application of HE4 into the diagnosis of RA. We found that the levels of HE4 were significantly elevated in patients with RA, particularly in patients with RAILD. Further, we showed that high levels of HE4 were an independent factor for identifying patients with RA-ILD. Given that biomarkers predicting RA patients at risk for ILD are currently lacking, our findings thus represent an important endeavor in risk stratification and clinical subset identification in patients with RA.

In this study, we found that the levels of HE4 were elevated in more than half of RA patients. When we further subgrouped RA patients according to HE4 status, we found that HE4-positive RA group had a higher percentage of RA-ILD compared to HE4negative RA group. This phenotype was also verified in a separate RA cohort. Recently, Zhang et al. reported that serum HE4 levels were significantly increased in patients with SSc-ILD compared to SSc-non-ILD, which was consistent with our findings (10). Further, we found that high levels of HE4 were independently associated with the presence of RA-ILD in a multivariate logistic regression analysis. Consistent with our results, Nishiyama et al. also showed that HE4 was a new biomarker to predict the prognosis of progressive fibrosing ILD (20). Taken together, these findings support a role of HE4 in risk stratification of ILD.

In this study, we showed a positive correlation between the levels of HE4 with the degree of lung impairment. Human epididymis protein 4 has been shown to be expressed in respiratory epithelium (9) and increased expression of HE4 was identified in lung biopsy from patients with cystic fibrosis 
TABLE 5 | Univariate and multivariate analyses for risk factors of rheumatoid arthritis (RA)-interstitial lung disease (ILD).

\begin{tabular}{|c|c|c|c|c|c|c|}
\hline \multirow[t]{2}{*}{ Variables } & \multicolumn{3}{|c|}{ Univariate analysis } & \multicolumn{3}{|c|}{ Multivariate analysis } \\
\hline & ILD+ $(n=48)$ & ILD- $(n=120)$ & $p$-value & ORs & $95 \% \mathrm{Cl}$ & $p$-value \\
\hline Gender, female & $36(75.0)$ & $111(92.5)$ & 0.002 & 0.275 & $0.085-0.893$ & 0.032 \\
\hline Age, year & $67(57,73)$ & $62(53,70)$ & 0.012 & 1.002 & $0.961-1.045$ & 0.992 \\
\hline Smoking status & $4(8.3)$ & $3(2.5)$ & 0.104 & & & \\
\hline Disease duration, year & $9(4,15)$ & $9(3,20)$ & 0.938 & & & \\
\hline SJC28 & $1(0,4)$ & $2(0,8)$ & 0.024 & & & \\
\hline TJC28 & $1(0,8)$ & $6(1,14)$ & 0.002 & & & \\
\hline $\mathrm{ESR} \geq 20 \mathrm{~mm} / \mathrm{h}$ & $31(64.6)$ & $86(71.7)$ & 0.367 & & & \\
\hline $\mathrm{CRP} \geq 8.0 \mathrm{mg} / \mathrm{L}$ & 27 (56.3) & 70 (58.3) & 0.805 & & & \\
\hline DAS28-ESR & $4.74(3.26,6.10)$ & $5.46(3.99,7.27)$ & 0.035 & 0.821 & $0.690-0.976$ & 0.026 \\
\hline High-RF, positive & $23(47.9)$ & $42(35.0)$ & 0.120 & & & \\
\hline High-anti-CCP antibody, positive & $34(70.8)$ & $67(55.8)$ & 0.073 & 1.410 & $0.602-3.301$ & 0.429 \\
\hline Leukopenia & $5(10.4)$ & $18(15.0)$ & 0.435 & & & \\
\hline Anemia & $23(47.9)$ & 71 (59.2) & 0.185 & & & \\
\hline Hyperglobulinemia & $10(20.8)$ & $18(15.0)$ & 0.359 & & & \\
\hline High HE4, positive & $34(70.8)$ & $27(22.5)$ & $<0.001$ & 9.080 & $3.481-23.682$ & $<0.001$ \\
\hline Sjögren's syndrome & $11(22.9)$ & 28 (23.3) & 0.954 & & & \\
\hline NSAIDs & $8(16.7)$ & $28(23.3)$ & 0.341 & & & \\
\hline Steroids & $18(37.5)$ & $32(26.7)$ & 0.165 & & & \\
\hline cDMARDs & 40 (83.3) & $92(76.7)$ & 0.341 & & & \\
\hline bDMARDs & $5(10.4)$ & 16 (13.3) & 0.606 & & & \\
\hline
\end{tabular}

Data are presented as median (Interquartile Range) and $n$ (\%). Statistical significance was determined by Mann-Whitney $U$ test, Chi-square ( $\chi 2$ ) test and Logistic regression analysis. Leukopenia was defined as white blood cell count $<4,000 / \mathrm{mm}^{3}$. Anemia was defined as hemoglobin levels $<110 \mathrm{~g} / \mathrm{L}$ for women. Hyperglobulinemia was defined as immunoglobulin G (IgG) level >16.8 g/L. High HE4 was defined as HE4 levels > 104.25 pmol/L based on Youden index. Anti-CCP, anti-cyclic citrullinated peptide antibody; bDMARDs, biological

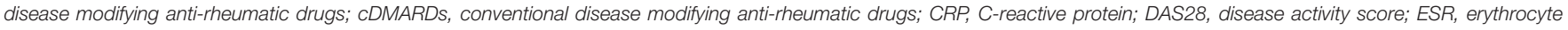
sedimentation rate; NSAIDs, non-steroid anti-inflammatory drugs; RF, rheumatoid factor; SJC, swollen joint counts in 28 joints; TJC, tender joint counts in 28 joints.

(CF) (17). A recent study found that elevated levels of HE4 was positively associated the degree of pulmonary dysfunction in patients with CF (19), which was consistent with our study. Further, the levels of HE4 inversely correlated with lung function improvement in CF patients after treatment, suggesting a diagnostic potential of HE4 in routine clinical and laboratory follow-up of CF treatment (21). Although it remains unclear whether HE4 is implicated in the pathogenesis of RA-ILD, LeBleu et al. have showed that HE4 can suppress the activity of multiple proteases, including serine proteases and matrix metalloproteinases, and specifically inhibits their capacity to degrade type I collagen, thereby promoting the development of kidney fibrosis (18). Further studies are needed to define the functional relevance of HE4 in the pathogenesis of RA-ILD.

Of interest, 25 (72\%) RA patients in the discovery cohort and $22(42 \%)$ RA patient in the validation cohort were positive for HE4, but did not have ILD, suggesting other RA-related factors may influenced the levels of HE4. Further studies investigating how the levels of HE4 were modulated in the context of RA will be of great importance. In addition, Krebs von den Lungen-6 (KL6 ) has been proposed as a potential biomarker in the diagnosis of ILD (22). It will be of great interest to assess whether combination of KL-6 with HE4 can improve the diagnostic value of each single biomarker.

Our study has a number of notable strengths. To the best of our knowledge, our study represents the first study investigating the clinical performance of $\mathrm{HE} 4$ in risk stratification of RA. Our findings thus expand our understanding of the clinical utility of $\mathrm{HE} 4$ in clinical practice, especially in rheumatoid diseases, such as RA. It should be noted, however, that our study has several limitations. First, it was a single-center study with a small RA cohort. Second, most participants of our study were female, which may result in analysis bias, as RA-ILD are more frequent in male patients. Third, patients of this study had long disease durations, and the levels of HE4 can't represent the onset status. Fourth, longitudinal examination of HE4 in patients with RA was missing. Further multi-center studies with a larger cohort comprising more male patients will be needed to corroborate our findings.

In summary, our findings showed a novel role of HE4 in RA risk stratification, suggesting that introducing HE4 to the current RA test panel (i.e., anti-CCP and RF) may provide additional diagnostic value to the current clinically available assays, especially in identifying RA patients for further RA-ILD workups, such as HRCT. Since RA-ILD represents a major complication responsible for morbidity and mortality in RA, this simple and highly reproducible biomarker, which has been already available in routine clinical practice in some countries, would be of great importance to risk-stratify RA patients for the performance of HRCT. 


\section{DATA AVAILABILITY STATEMENT}

The raw data supporting the conclusions of this article will be made available by the authors, without undue reservation.

\section{ETHICS STATEMENT}

The studies involving human participants were reviewed and approved by the Ethical Committee of PKUPH (Protocol number: 2019PHB244). The patients/participants provided their written informed consent to participate in this study.

\section{REFERENCES}

1. Smolen JS. Rheumatoid arthritis primer - behind the scenes. Nat Rev Dis Primers. (2020) 6:32. doi: 10.1038/s41572-020-0168-y

2. Alunno A, Gerli R, Giacomelli R, Carubbi F. Clinical, epidemiological, and histopathological features of respiratory involvement in rheumatoid arthritis. Biomed Res Int. (2017) 2017:7915340. doi: 10.1155/2017/7915340

3. Chen RX, Zhao LD, Xiao XY, Song L, Du HY, Xu ZJ, et al. Distinctive clinical characteristics and outcome of ild-onset rheumatoid arthritis and ACPApositive ILD: a longitudinal cohort of 282 cases. Clin Rev Allergy Immunol. (2020) 60:46-54. doi: 10.1007/s12016-020-08819-0

4. Sparks JA, Chang SC, Liao KP, Lu B, Fine AR, Solomon DH, et al. Rheumatoid arthritis and mortality among women during 36 years of prospective followup: results from the nurses' health study. Arthritis Care Res (Hoboken). (2016) 68:753-62. doi: 10.1002/acr.22752

5. England BR, Hershberger, D. Management issues in rheumatoid arthritisassociated interstitial lung disease. Curr Opin Rheumatol. (2020) 32:25563. doi: 10.1097/BOR.0000000000000703

6. Raimundo K, Solomon JJ, Olson AL, Kong AM, Cole AL, Fischer A, et al. Rheumatoid arthritis-interstitial lung disease in the United States: prevalence, incidence, and healthcare costs and mortality. J Rheumatol. (2019) 46:3609. doi: 10.3899/jrheum.171315

7. Olson AL, Swigris JJ, Sprunger DB, Fischer A, Fernandez-Perez ER, Solomon J, et al. Rheumatoid arthritis-interstitial lung disease-associated mortality. Am J Respir Crit Care Med. (2011) 183:372-8. doi: 10.1164/rccm.201004-0622OC

8. Ferraro S, Panteghini M. Making new biomarkers a reality: the case of serum human epididymis protein 4. Clin Chem Lab Med. (2019) 57:128494. doi: 10.1515/cclm-2018-1111

9. Galgano MT, Hampton GM, Frierson HF, Jr. Comprehensive analysis of HE4 expression in normal and malignant human tissues. Mod Pathol. (2006) 19:847-53. doi: 10.1038/modpathol.3800612

10. Zhang $\mathrm{M}$, Zhang L, Linning E, Xu K, Wang XF, Zhang B, et al. Increased levels of HE4 (WFDC2) in systemic sclerosis: a novel biomarker reflecting interstitial lung disease severity? Ther Adv Chronic Dis. (2020) 11:2040622320956420. doi: 10.1177/2040622320956420

11. Chen J, Sun F, Bao H, Liang L, Zhan M, Yao H, et al. Elevated serum human epididymis protein 4 is associated with disease activity and systemic involvements in primary Sjogren's syndrome. Front Immunol. (2021) 12:670642. doi: 10.3389/fimmu.2021.670642

12. Aletaha D, Neogi T, Silman AJ, Funovits J, Felson DT, Bingham CO III, et al. 2010 Rheumatoid arthritis classification criteria: an American College of Rheumatology/European League Against Rheumatism collaborative initiative. Arthritis Rheum. (2010) 62:2569-81. doi: 10.1002/art.27584

13. Prevoo ML, van 't Hof MA, Kuper $H H$, van Leeuwen MA, van de Putte LB, van Riel PL. Modified disease activity scores that include twentyeight-joint counts development and validation in a prospective longitudinal study of patients with rheumatoid arthritis. Arthritis Rheum. (1995) 38:448. doi: 10.1002/art.1780380107

14. Liu Y, Liu C, Li L, Zhang F, Li Y, Zhang S. High levels of antibodies to citrullinated alpha-enolase peptide-1 (CEP-1) identify erosions and interstitial

\section{AUTHOR CONTRIBUTIONS}

LL, JC, and CD: experiments, data acquisition, and data analysis. $\mathrm{MZ}, \mathrm{HB}, \mathrm{CX}$, and $\mathrm{CF}$ : experiments and data acquisition. YL: study design, data analysis, and manuscript preparation. All authors contributed to the article and approved the submitted version.

\section{FUNDING}

This work was supported in part by grants from National Natural Science Foundation of China, grant no. 81971521.

lung disease (ILD) in a Chinese rheumatoid arthritis cohort. Clin Immunol. (2019) 200:10-5. doi: 10.1016/j.clim.2019.01.001

15. Standardization of spirometry, 1994 update. American Thoracic Society. Am J Respir Crit Care Med. (1995) 152:1107-36. doi: 10.1164/ajrccm.152.3. 7663792

16. Goh NS, Desai SR, Veeraraghavan S, Hansell DM, Copley SJ, Maher TM, et al. Interstitial lung disease in systemic sclerosis: a simple staging system. Am J Respir Crit Care Med. (2008) 177:1248-54. doi: 10.1164/rccm.2007 06-877OC

17. Bingle L, Cross SS, High AS, Wallace WA, Rassl D, Yuan G, et al. WFDC2 (HE4): a potential role in the innate immunity of the oral cavity and respiratory tract and the development of adenocarcinomas of the lung. Respir Res. (2006) 7:61. doi: 10.1186/1465-9921-7-61

18. LeBleu VS, Teng Y, O’Connell JT, Charytan D, Muller GA, Muller CA, et al. Identification of human epididymis protein-4 as a fibroblast-derived mediator of fibrosis. Nat Med. (2013) 19:227-31. doi: 10.1038/nm.2989

19. Nagy B Jr, Nagy B, Fila L, Clarke LA, Gonczy F, Bede O, et al. human epididymis protein 4: a novel serum inflammatory biomarker in cystic fibrosis. Chest. (2016) 150:661-72. doi: 10.1016/j.chest.2016.04.006

20. Nishiyama N, Masuo M, Nukui Y, Tateishi T, Kishino M, Tateishi U, et al. Human epididymis protein 4 is a new biomarker to predict the prognosis of progressive fibrosing interstitial lung disease. Respir Investig. (2021) 59:908. doi: 10.1016/j.resinv.2020.08.002

21. Nagy B Jr, Bene Z, Fejes Z, Heltshe SL, Reid D, Ronan NJ, et al. Human epididymis protein 4 (HE4) levels inversely correlate with lung function improvement (delta FEV1) in cystic fibrosis patients receiving ivacaftor treatment. J Cyst Fibrosis. (2019) 18:271-7. doi: 10.1016/j.jcf.2018.08.013

22. Wang $\mathrm{Y}$, Chen $\mathrm{S}$, Lin J, Xie X, Hu S, Lin Q, et al. Lung ultrasound Blines and serum KL-6 correlate with the severity of idiopathic inflammatory myositis-associated interstitial lung disease. Rheumatology (Oxford). (2020) 59:2024-9. doi: 10.1093/rheumatology/kez571

Conflict of Interest: The authors declare that the research was conducted in the absence of any commercial or financial relationships that could be construed as a potential conflict of interest.

Publisher's Note: All claims expressed in this article are solely those of the authors and do not necessarily represent those of their affiliated organizations, or those of the publisher, the editors and the reviewers. Any product that may be evaluated in this article, or claim that may be made by its manufacturer, is not guaranteed or endorsed by the publisher.

Copyright (c) 2021 Liang, Chen, Di, Zhan, Bao, Xia, Fan and Liu. This is an openaccess article distributed under the terms of the Creative Commons Attribution License (CC BY). The use, distribution or reproduction in other forums is permitted, provided the original author(s) and the copyright owner(s) are credited and that the original publication in this journal is cited, in accordance with accepted academic practice. No use, distribution or reproduction is permitted which does not comply with these terms. 